\title{
Change Trend of Plasma D-Dimer Level and Its Clinical Value in Patients with Acute Lower Limb Ischemia Treated by Catheter-Directed Thrombolysis
}

Xiaochun Liu

Ganzhou People's Hospital

Hailiang Xie

Ganzhou People's Hospital

Guofu Zheng

Ganzhou People's Hospital

Yuanfei Liu ( $\sim$ liuyuanfeigzsrmyy@163.com )

Ganzhou People's Hospital

\section{Research Article}

Keywords: Acute lower limb ischemia, Catheter-directed thrombolysis, D-dimer, Change trend

Posted Date: January 13th, 2021

DOl: https://doi.org/10.21203/rs.3.rs-143092/v1

License: (c) (i) This work is licensed under a Creative Commons Attribution 4.0 International License.

Read Full License 


\section{Abstract}

To investigate the correlation between plasma D-dimer levels before and after catheter-directed thrombolysis (CDT) in acute lower limb ischemia (ALI) and lower limb arterial recanalization and the clinical value of the change trend of plasma D-dimer. A retrospective review of all patients who received CDT for the treatment of ALI between January 2013 and December 2019 was carried out. The change trend of plasma D-dimer of the patients during thrombolysis was statistically analyzed using repeated measures ANOVA. A total of 150 patients were included in the analysis. Among them, 3 days of CDT was ineffective in 41 cases, effective in 33 cases and markedly effective in 76 cases. The plasma D-dimer levels of the patients were significantly different between pre- and post-thrombolysis $(F=23.856, P=$ 0.000). There was an interaction effect between the measured time factors and thrombolysis efficacy $(F=13.991, P=0.000)$. The change trends of plasma $D$-dimer levels in the three groups were statistically significant $(F=3.246, P=0042)$. The plasma $D$-dimer level changes regularly during CDT for ALI. We can predict the efficacy of CDT and guide adjustments of the therapeutic regimen according to the trend of Ddimer changes during thrombolysis to obtain better clinical effects.

\section{Introduction}

Acute lower limb ischemia (ALI) is caused by a reduction in arterial perfusion in the limb due to embolic migration or local thrombosis. In addition to the risk of limb damage or loss, it can cause life-threatening complications related to metabolic conditions triggered throughout the body. ALI should be treated urgently ${ }^{1}$. Catheter-directed thrombolysis (CDT) has been used for the treatment of ALI for over 20 years ${ }^{2-}$

${ }^{4}$. Thrombolytic therapy can cause significant changes in plasma D-dimer ${ }^{5-7}$. D-dimer is a soluble fibrin degradation product that is the result of the orderly breakdown of thrombi by the fibrinolysis system. Its level is commonly used in the clinical diagnosis and treatment evaluation of venous thromboembolic $(\mathrm{VTE})^{8}$. Thrombolytic efficacy can be evaluated by monitoring plasma D-dimer levels during CDT in patients with deep vein thrombosis ${ }^{9}$. Before and after thrombolysis, the changes in plasma D-dimer levels are also considered to be significantly correlated with coronary recanalization, which could be used to predict the thrombolysis efficacy after acute myocardial infarction ${ }^{6,7}$.

The correlation between plasma D-dimer level and reperfusion has not yet been clarified in CDT applied for patients with ALI. Therefore, the purpose of this study was to investigate the correlation between the change trends of plasma D-dimer levels before and after CDT in ALI and lower limb arterial recanalization and whether the plasma D-dimer levels could predict the efficacy of thrombolysis.

\section{Methods}

\section{Ethics and consent statement}

This retrospective cohort study was approved by the Medical Ethics Committee of Ganzhou People's Loading [MathJax]/jax/output/CommonHTML/fonts/TeX/fontdata.js nce with the approved guidelines. Written 
informed consent was signed by the patients' direct relatives.

\section{Study population}

We retrospectively analyzed consecutive patients with ALI treated with CDT at Ganzhou People's Hospital from January 2013 to December 2019. Inclusion criteria: Patients receiving CDT intravascular treatment for ALI who were treated for at least 3 days. The exclusion criteria were as follows: (1) nonacute lower limb ischemia; (2) incomplete data; (3) patients receiving mechanical thromboembolism treatment; and (4) CDC duration shorter than 3 days.

The data of interest were collected through the electronic medical record system, focusing on plasma Ddimer levels during thrombolysis. In addition, according to the research needs, we collected the basic information of the eligible patients, including gender, age, alcohol consumption, smoking, degree of ischemia (Rutherford classification ${ }^{10}$ ), artery occlusion site, length of the thrombolytic catheter, and urokinase usage. Comorbidities of the patients were also collected, including hypertension, diabetes mellitus, atrial fibrillation, ischemic heart disease, cerebrovascular disease, renal insufficiency, chronic obstructive pulmonary disease, hyperlipidemia, and liver function.

\section{CDT procedure}

The CDT procedure involves placing a multihole infusion catheter (Unifuse, AngioDynamics) into the thrombosis occlusion arterial segment of the lower limb under the guidance of digital subtraction angiography (DSA) and continuously pumping urokinase through the catheter. Urokinase is used at a dose of 30,000 to $50,000 \mathrm{U} / \mathrm{h}$ and then adjusted in accordance with the plasma fibrinogen levels.

Thrombolysis will be stopped if the thrombus is completely dissolved or if the patient's plasma fibrin level is $<1 \mathrm{~g} / \mathrm{L}$ or complications of severe hemorrhage (such as bleeding at the puncture site or other sites) occur after thrombolysis.

\section{Plasma D-dimer level monitoring}

Blood samples to measure circulating levels of plasma D-dimer were collected from the peripheral blood on admission, and serial samples were then collected at $8,16,24,32,40,48,56,64$, and $72 \mathrm{~h}$ post-CDT. At least 10 repeated measurements of plasma D-dimer were performed in each case.

\section{Grouping}

For the purpose of this analysis, the patients were further divided into three subgroups depending on the immediate imaging results of the DSA images taken on the third day of CDT and limb distal arterial 


\section{Markedly effective group:}

DSA images showed occluded segment unobstruction; pulsation of the dorsal foot artery and/or posterior tibial artery were palpable; and the lower extremity pain completely disappeared.

\section{Effective group:}

DSA images showed that the occluded segment was completely or partially unobstructed. The dorsal foot artery and/or posterior tibial artery pulses were not detectable, but the skin temperature of the lower extremity was warm, and the pain was relieved.

\section{Ineffective group:}

DSA images showed the occluded segment was still obstructed; the dorsal foot artery and/or posterior tibial artery pulses were not palpable; and the lower extremity pain did not improve.

\section{Statistical analysis}

SPSS software package version 24.0 (IBM, Armonk, NY, USA) was used for data management and statistical analysis. The continuous data of the three groups were described by means \pm standard deviations (SDs), nonparametric data were expressed as medians (interquartile ranges), and categorical data were presented as percentages. The ordinal data of the three groups were analyzed by the KruskalWallis test. Repeated measures ANOVA was used to analyze the change trends in the D-dimer levels among the three groups. All tests were two-sided with a significance level of 0.05 .

\section{Results}

\section{General data of the patients.}

From January 2013 to December 2019, 325 patients with lower limb ischemia received CDT treatment in our hospital. Of them, 61 patients had non-ALI, 21 patients had incomplete information (no repeated measurement data of D-dimer), 35 patients underwent mechanical thrombus aspiration at the same time, and 58 patients continued thrombolysis for $<3$ days (patients who had the thrombolytic catheter removed early because they had achieved good thrombolytic results or were unable to adhere to CDT due to complications such as severe bleeding), so the remaining 150 patients who met the inclusion criteria were included in the analysis (Fig. 1).

The average age was 76.1 (56-79) years, and 81 were males. The ischemic level of Rutherford Illb accounted for the highest proportion (92 cases, $61.3 \%$ ). The most common site of occlusion was the Loading [MathJax]/jax/output/CommonHTML/fonts/TeX/fontdata.js -DT was ineffective in 41 cases (27.3\%) and 
effective in 33 cases and markedly effective in 76 cases, accounting for $73.7 \%$. The patients were divided into three groups according to their outcome, and the baseline characteristics of the three groups are shown in Table 1. There were no statistically significant differences among the three groups for the degree of ischemia, occlusion site, type of thrombolytic catheter, or amount of urokinase.

\section{Analysis of variance of repeated measures data of plasma D-dimer levels in the 3 groups}

The plasma D-dimer levels of the three groups with different thrombolytic efficacies are shown in Table 2. The plasma $D$-dimer levels were significantly different pre- and post-thrombolysis $(F=23.856, P=0.000)$. The same was true in each group, with $F$ values of $42.452,58.01$, and 124.12 , respectively, $P=0.000$. No difference was found in plasma D-dimer levels among the three groups before thrombolysis ( $x^{2}=2.656, \mathrm{P}=0.265$ ) (Kruskal-Wallis test). There was an interaction effect between the measured time factors and the thrombolysis results $(F=13.991, P=0.000)$. The change trends of plasma $D$-dimer levels in the three groups were statistically significant $(F=3.246, P=0042)$. The trend chart (Fig. 2$)$ showed that the plasma D-dimer level in all three groups increased at the beginning and then decreased after thrombolysis. The d-dimer level in the markedly effective group increased rapidly at the beginning of thrombolysis, reached its peak 16 hours later, and then decreased quickly to the preoperative level. The $d-$ dimer of the ineffective and effective groups increased slowly after beginning thrombolysis, peaked 24 hours later, and then slowly declined. In the ineffective group, the plasma D-dimer level increased again 48 hours after beginning thrombolysis and had another peak.

\section{Discussion}

This study indicated that the relationship between the change trend of plasma D-dimer levels before and after CDT treatment and thrombolytic outcomes for ALI. By analyzing the plasma D-dimer change trends, we can predict the reperfusion of ischemic lower limbs after thrombolytic therapy and know the results of CDT in a timely manner. This has rarely been reported in previous studies.

ALI is caused by a sudden interruption of the main blood perfusion of the lower limb for various reasons, and it usually requires immediate revascularization. CDT has become one of the most commonly used methods for the treatment of ALI ${ }^{11,12}$. After thrombolytic therapy, the vascular stenosis, occlusion and other lesions can be treated simultaneously with interventional therapy, which improves the short-term and long-term patency rate of such occlusive lesions and reduces the need for open surgery in most of these patients ${ }^{4}$.

D-dimer is the degradation product of cross-linked fibrin, and its plasma level reflects the formation and degradation of fibrin. D-dimer is considered a useful biomarker because of its potential to identify individuals in a high coagulation state. D-dimer is often associated with thrombi, and its elevation should 
D-dimer test is negative, the diagnosis of acute VTE can be ruled out; if the plasma D-dimer test is positive, the diagnosis of VTE can be confirmed by the symptoms and an ultrasound examination ${ }^{8}$. There have also been many relevant studies in some cardiovascular diseases, such as atrial fibrillation ${ }^{18-20}$, coronary heart disease ${ }^{13,21,22}$, stroke $\mathrm{e}^{23,24}$ and aortic dissection ${ }^{25-27}$, and D-dimer can even be used as a predictor of the diagnosis and prognosis of such diseases ${ }^{28-31}$.

D-dimer levels are lower in the circulation in healthy individuals and higher in the presence of thrombi ${ }^{8}$. During the CDT process, the fibrin in the thrombus degrades, and D-dimer is produced. These dissolved substances are continuously released into the blood, causing the plasma D-dimer content to change constantly $9,32,33$. The D-dimer level increases continuously during thrombolysis and is positively correlated with the degree of thrombolysis ${ }^{5}$. D-dimer has a half-life of approximately 8 hours until it is cleared by the kidney and reticuloendothelial system ${ }^{34}$. In the later stage of thrombolysis, with the gradual opening of the occluded vessels, the thrombus load in the vessels decreases, and the D-dimer content in the blood decreases continuously until it returns to the prethrombolysis level. Monitoring the changes in plasma D-dimer content pre- and posttreatment is also often used to evaluate the therapeutic effect $^{7,32,33,35}$. Davies, J. et al. analyzed systemic inflammatory response syndrome following catheterdirected thrombolysis of acute iliofemoral deep venous thrombosis and found that plasma D-dimer increased from $1465 \mathrm{ng} / \mathrm{ml}$ to $182,835 \mathrm{ng} / \mathrm{ml}$ after 48 hours of thrombolysis and then decreased to the prethrombolysis level 36 hours later. As a result of this presentation, thrombus occlusion of the iliofemoral vein had restored patency after CDT treatment ${ }^{9}$. Cakar, M. A. et al. analyzed D-dimer levels preand post-thrombolysis in 186 patients with acute coronary syndrome treated with intravenous tissue-type plasminogen activator $(100 \mathrm{mg})$ or streptokinase $(1,500000 \mathrm{U})$ and found that D-dimer levels were markedly high after thrombolytic therapy versus before $(155 \mathrm{mg} / \mathrm{dl}, 362 \mathrm{mg} / \mathrm{dl}, \mathrm{P}<0.005)^{6}$.

For the convenience of analysis, we grouped our patients according to the results of thrombolysis. The change trends of D-dimer in the three groups during thrombolysis are shown in Fig. 2. During thrombolytic therapy, the lower limb arteries of patients whose plasma D-dimer level rose rapidly, reached its peak, and then rapidly dropped to the preoperative level obtained complete recanalization. The lower limb arteries of patients whose plasma D-dimer level rose slowly, then reached its peak slowly or had two peaks, and then dropped slowly to the preoperative level might achieve partial recanalization or remain occluded.

Accordingly, we believe that after CDT treatment of ALI, if the plasma D-dimer levels show a significant increase within a short period of time and then a rapid decline without a rebound, this suggests that the thrombolytic therapy was effective. If the plasma D-dimer continues to rise slowly and decline slowly, this suggests that the thrombolytic catheter may not be insufficient contact with the thrombus or the thrombolytic drug dose is insufficient, which requires appropriate adjustments. If the D-dimer exhibits multiple peaks, this may be due to the presence of a new thrombus, unobstructed vascular outflow tract or occlusion of the artery by the old thrombus, which should be solved by combining CDT with other 
ineffective treatment of CDT for the patients in this study, and then corresponding measures were taken, such as adjusting the location of the thrombolytic catheter, increasing the time and amount of the thrombolytic agent, or combining the CDT with percutaneous transluminal angiography (PTA)/stent implantation. These additional measures improved the blood supply of the ischemic limbs, avoiding gangrene. As a result, the limb salvage rate of patients with ALI was improved.

Several limitations of this analysis should be acknowledged. In this study, patients with CDT thrombolysis for 1-2 days were excluded because these patients did not have 3 days of complete Ddimer data, which reduced the sample size of our study. In addition, the grouping in this study was based on the recovery of the lower limb blood supply of patients on the third day of CDT. In fact, patients with a poor CDT response were still receiving treatment after 3 days, such as continued CDT treatment or a combination of other treatments, so the final clinical results for all of the patients were not provided in this study. In addition, CDT therapy for non-ALI is also widely used in clinical practice ${ }^{36,37}$, but the lesions in these patients are not mainly thrombotic and often require PTA and/or stent implantation, so they were excluded from this study. Therefore, a multicenter, prospective, randomized controlled study may be the best way to further understand the trends and clinical significance of plasma D-dimer changes during CDT in patients with ALI.

\section{Conclusion}

In conclusion, the plasma D-dimer level changes regularly during CDT for ALI. Occluded arteries may be recanalized in patients whose D-dimer appears to rise rapidly during CDT, peaks, and then rapidly declines to pretreatment levels. Therefore, we can predict the results of CDT and guide adjustment of the therapeutic regimen according to the trends of $D$-dimer changes during thrombolysis to obtain better clinical effects and allow more ischemic limbs to be salvaged.

\section{Declarations}

\section{Author Contributions}

Y.F.L. conceptualized and designed the study. X.C.L., G.F.Z., and H.L.X. performed and supervised data collection. X.C.L. and G.F.Z. contributed in data analysis. X.C.L. and G.F.Z. prepared the first draft of the paper. All the authors contributed in revision of the manuscript. All the authors have read and approved the final manuscript.

\section{Additional Information}

Supplementary information accompanies this paper at 
The author(s) declare no competing interests.

\section{References}

1. Creager, M. A., Kaufman, J. A. \& Conte, M. S. Acute Limb Ischemia. N Engl J Med. 366, 2198-2206 https://doi.org/10.1056/NEJMcp1006054 (2012).

2. Theodoridis, P. G. et al. Thrombolysis in Acute Lower Limb Ischemia: Review of the Current Literature. Ann Vasc Surg. 52, 255-262 https://doi.org/10.1016/j.avsg.2018.02.030 (2018).

3. Ouriel, K., Veith, F. J. \& Sasahara, A. A. A comparison of recombinant urokinase with vascular surgery as initial treatment for acute arterial occlusion of the legs. Thrombolysis or Peripheral Arterial Surgery (TOPAS) Investigators. N Engl J Med. 338, 1105-1111 https://doi.org/10.1056/NEJM199804163381603 (1998).

4. Grip, O., Wanhainen, A., Acosta, S. \& Bjorck, M. Long-term Outcome after Thrombolysis for Acute Lower Limb Ischaemia. Eur J Vasc Endovasc Surg. 53, 853-861 https://doi.org/10.1016/j.ejvs.2017.02.003 (2017).

5. Liu, M. \& Zhang, F. Administration routes affect thrombolytic effect of catheter-directed thrombolysis with pro-urokinase in treating deep vein thrombosis. Ann Trans/ Med. 6, 322 https://doi.org/10.21037/atm.2018.08.01 (2018).

6. Cakar, M. A. et al. Correlation between D-dimer levels and coronary artery reperfusion in acute myocardial infarction patients after thrombolytic treatment. Blood Coagul Fibrinolysis. 24, 608-612 https://doi.org/10.1097/MBC.0b013e328360a53f (2013).

7. B, B. et al. Relation of plasma D-dimer concentrations to coronary artery reperfusion before and after thrombolytic treatment in patients with acute myocardial infarction. Am J Cardiol. 63, 1179-1184 (1989).

8. Weitz, J. I., Fredenburgh, J. C. \& Eikelboom, J. W. A Test in Context: D-Dimer. J Am Coll Cardiol. 70, 2411-2420 https://doi.org/10.1016/j.jacc.2017.09.024 (2017).

9. Davies, J., Tippet, R., Watson, A. \& Metcalfe, J. Systemic inflammatory response syndrome following catheter-directed thrombolysis of acute iliofemoral deep venous thrombosis. Phlebology. 32, 61-65 https://doi.org/10.1177/0268355515627261 (2017).

10. Rutherford, R. B. Clinical staging of acute limb ischemia as the basis for choice of revascularization method: when and how to intervene. Semin Vasc Surg. 22, 5-9 https://doi.org/10.1053/j.semvascsurg.2008.12.003 (2009).

11. Lian, W. S. et al. Efficacy of intra-arterial catheter-directed thrombolysis for popliteal and infrapopliteal acute limb ischemia. J Vasc Surg. 71, 141-148 https://doi.org/10.1016/j.jvs.2019.03.081 (2020).

12. Grip, O. et al. Outcome and complications after intra-arterial thrombolysis for lower limb ischaemia with or without continuous heparin infusion. Br J Surg. 101, 1105-1112 httns //dni ora/10 10n2/his 9.579 (2014) 
13. Gong, P. et al. Plasma d-Dimer as a Useful Marker Predicts Severity of Atherosclerotic Lesion and Short-Term Outcome in Patients With Coronary Artery Disease. Clin Appl Thromb Hemost. 22, 633640 https://doi.org/10.1177/1076029616634885 (2016).

14. Narang, J., Nowacki, A. S., Seballos, S. S., Wang, P. R. \& Mace, S. E. D-dimer can help differentiate suspected pulmonary embolism patients that require anti-coagulation. Am J Emerg Med. https://doi.org/10.1016/j.ajem.2020.08.086 (2020).

15. Yucel, O., Yucel, H. \& Zorlu, A. D-dimer is a predictor of cardiovascular death, and new-onset atrial fibrillation in patients with systolic heart failure. Int J Cardiol. 247, 39 https://doi.org/10.1016/j.ijcard.2017.04.065 (2017).

16. Tang, N. et al. Combined measurement of factor XIII and D-dimer is helpful for differential diagnosis in patients with suspected pulmonary embolism. Clin Chem Lab Med. 55, 1948-1953 https://doi.org/10.1515/cclm-2017-0072 (2017).

17. Cohen, A. T. et al. D-dimer as a predictor of venous thromboembolism in acutely ill, hospitalized patients: a subanalysis of the randomized controlled MAGELLAN trial. J Thromb Haemost. 12, 479487 https://doi.org/10.1111/jth.12515 (2014).

18. Hijazi, Z., Oldgren, J., Siegbahn, A. \& Wallentin, L. Application of Biomarkers for Risk Stratification in Patients with Atrial Fibrillation. Clin Chem. 63, 152-164 https://doi.org/10.1373/clinchem.2016.255182 (2017).

19. Siegbahn, A. et al. D-dimer and factor VIla in atrial fibrillation - prognostic values for cardiovascular events and effects of anticoagulation therapy. A RE-LY substudy. Thromb Haemost. 115, 921-930 https://doi.org/10.1160/TH15-07-0529 (2016).

20. Ruff, C. T. et al. Cardiovascular Biomarker Score and Clinical Outcomes in Patients With Atrial Fibrillation: A Subanalysis of the ENGAGE AF-TIMI 48 Randomized Clinical Trial. JAMA Cardiol. 1, 999-1006 https://doi.org/10.1001/jamacardio.2016.3311 (2016).

21. Simes, J. et al. D-Dimer Predicts Long-Term Cause-Specific Mortality, Cardiovascular Events, and Cancer in Patients With Stable Coronary Heart Disease: LIPID Study. Circulation. 138, 712-723 https://doi.org/10.1161/CIRCULATIONAHA.117.029901 (2018).

22. Folsom, A. R. et al. Associations of factor VIIIc, D-dimer, and plasmin-antiplasmin with incident cardiovascular disease and all-cause mortality. Am J Hematol. 84, 349-353 https://doi.org/10.1002/ajh.21429 (2009).

23. Folsom, A. R., Gottesman, R. F., Appiah, D., Shahar, E. \& Mosley, T. H. Plasma d-Dimer and Incident Ischemic Stroke and Coronary Heart Disease: The Atherosclerosis Risk in Communities Study. Stroke. 47, 18-23 https://doi.org/10.1161/STROKEAHA.115.011035 (2016).

24. Wang, Y. et al. The correlation of D-dimer levels with patient outcomes in acute ischemic cerebrovascular disease complicating coronary heart disease. Neurol Res. 38, 524-532 https://doi.org/10.1080/01616412.2016.1187829 (2016).

25. Watanabe, H. et al. Diagnostic test accuracy of D-dimer for acute aortic syndrome: systematic review 
https://doi.org/10.1038/srep26893 (2016).

26. Asha, S. E. \& Miers, J. W. A Systematic Review and Meta-analysis of D-dimer as a Rule-out Test for Suspected Acute Aortic Dissection. Ann Emerg Med. 66, 368-378 https://doi.org/10.1016/j.annemergmed.2015.02.013 (2015).

27. Yuan, S. M., Shi, Y. H., Wang, J. J., Lu, F. Q. \& Gao, S. Elevated plasma D-dimer and hypersensitive Creactive protein levels may indicate aortic disorders. Rev Bras Cir Cardiovasc. 26, 573-581 https://doi.org/10.5935/1678-9741.20110047 (2011).

28. Wang, J., Wu, X. Y., Liang, Y. \& Guo, W. Predictive value of the Wells score combined with D-dimer level in identifying acute pulmonary embolism in patients with coronary heart disease with chest pain. Chin Med J (Engl). 133, 2253-2255 https://doi.org/10.1097/CM9.0000000000000988 (2020).

29. Mjelva, O. R. et al. Long-term prognostic utility of pentraxin 3 and D-dimer as compared to highsensitivity C-reactive protein and B-type natriuretic peptide in suspected acute coronary syndrome. Eur J Prev Cardiol. 23, 1130-1140 https://doi.org/10.1177/2047487315619733 (2016).

30. Zhang, X., Wang, S., Sun, L., Fang, S. \& Yu, B. Prognostic value of D-dimer in acute myocardial infarction complicated by heart failure with preserved ejection fraction. ESC Heart Fail. https://doi.org/10.1002/ehf2.13027 (2020).

31. Kremers, B. et al. Plasma Biomarkers to Predict Cardiovascular Outcome in Patients With Peripheral Artery Disease: A Systematic Review and Meta-Analysis. Arterioscler Thromb Vasc Biol. 40, 20182032 https://doi.org/10.1161/ATVBAHA.120.314774 (2020).

32. Takamura, T. A. et al. Circulating malondialdehyde-modified low-density lipoprotein (MDA-LDL) as a novel predictor of clinical outcome after endovascular therapy in patients with peripheral artery disease (PAD). Atherosclerosis. 263, 192-197 https://doi.org/10.1016/j.atherosclerosis.2017.06.029 (2017).

33. Hsu, P. J. et al. High Plasma D-Dimer Indicates Unfavorable Outcome of Acute Ischemic Stroke Patients Receiving Intravenous Thrombolysis. Cerebrovasc Dis. 42, 117-121 https://doi.org/10.1159/000445037 (2016).

34. Hager, K. \& Platt, D. Fibrin degeneration product concentrations (D-dimers) in the course of ageing. Gerontology. 41, 159-165 https://doi.org/10.1159/000213677 (1995).

35. Engelberger, R. P. et al. Enhanced Thrombolysis by Ultrasound-Assisted Catheter-Directed Thrombolysis and Microbubbles in an In Vitro Model of lliofemoral Deep Vein Thrombosis. Thromb Haemost. 119, 1094-1101 https://doi.org/10.1055/s-0039-1688973 (2019).

36. Lukasiewicz, A., Flisinski, P. \& Lichota, W. Catheter-directed thrombolysis is not limited to acute limb ischemia treatment: experience from a division of vascular surgery. J Cardiovasc Surg (Torino). 61, 200-207 https://doi.org/10.23736/S0021-9509.17.10023-6 (2020).

37. Gunes, Y., Sincer, I. \& Erdal, E. Catheter-directed intra-arterial thrombolysis for lower extremity arterial occlusions. Anatol J Cardiol. 22, 54-59 https://doi.org/10.14744/AnatolJCardiol.2019.63296 (2019). 


\section{Tables}

Due to technical limitations, tables are only available as a download in the Supplemental Files section.

\section{Figures}

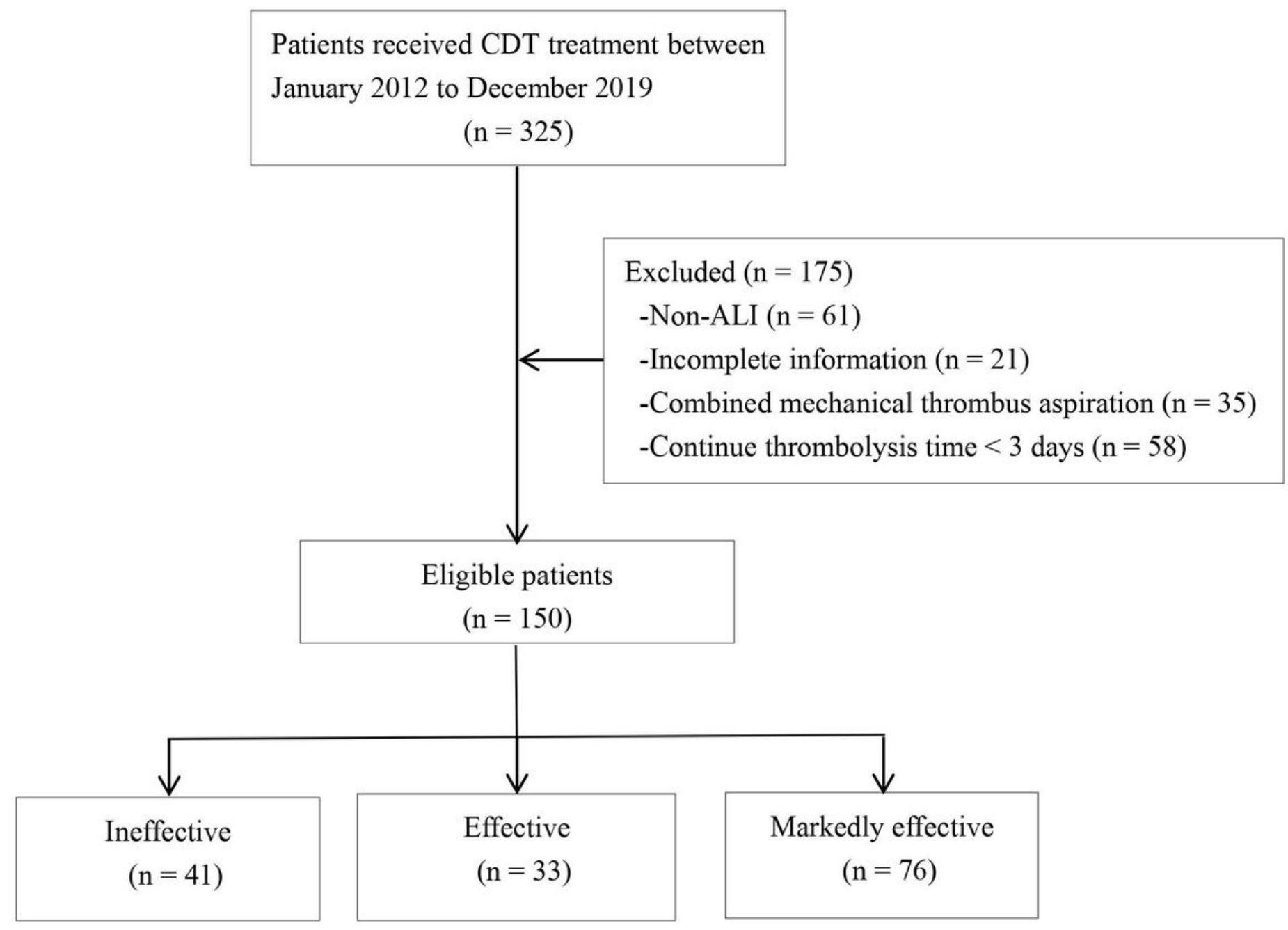

Figure 1

Patient Flowchart 


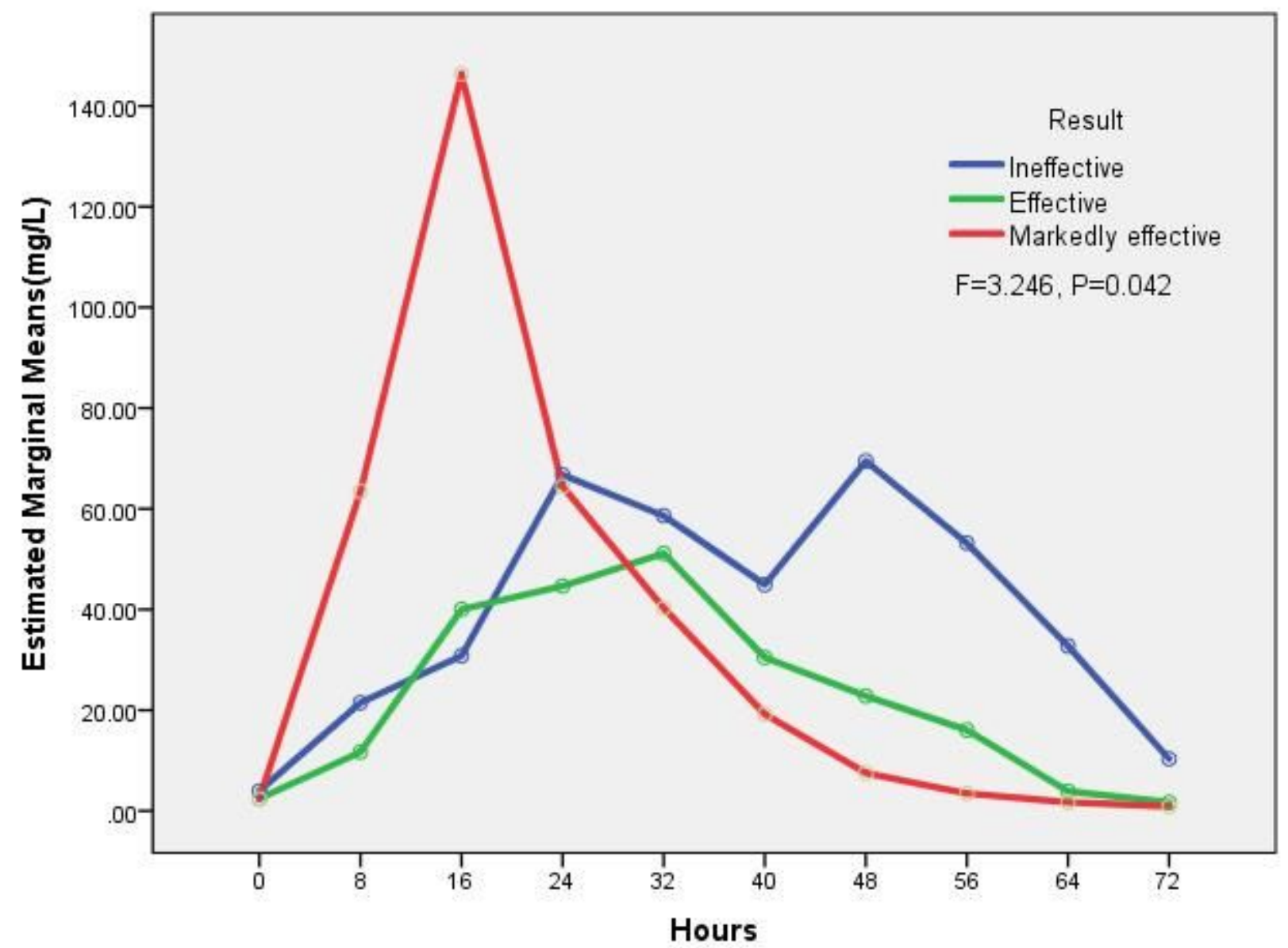

Figure 2

The cumulative rate of ulcer healing

\section{Supplementary Files}

This is a list of supplementary files associated with this preprint. Click to download.

- Table1.Baselinecharacteristicsofthepatients.docx

- Table2.AnalysisofvarianceofrepeatedmeasuredataofplasmaDdimerlevels.docx 